\title{
Transatlantica
}

Revue d'études américaines. American Studies Journal

\section{Un militantisme aux allures de mission impossible : l'exemple du lobby républicain pro-choice, la Republican Majority for Choice}

\section{Françoise Coste}

\section{(2) OpenEdition}

\section{Journals}

Édition électronique

URL : http://journals.openedition.org/transatlantica/3803

DOI : $10.4000 /$ transatlantica.3803

ISSN : $1765-2766$

Éditeur

AFEA

Référence électronique

Françoise Coste, « Un militantisme aux allures de mission impossible : l'exemple du lobby républicain pro-choice, la Republican Majority for Choice », Transatlantica [En ligne], 1 | 2008, mis en ligne le 12 juin 2008, consulté le 29 avril 2021. URL : http://journals.openedition.org/transatlantica/3803 ; DOI : https://doi.org/10.4000/transatlantica.3803

Ce document a été généré automatiquement le 29 avril 2021.

\section{(c) (1) $\odot$}

Transatlantica - Revue d'études américaines est mis à disposition selon les termes de la licence Creative Commons Attribution - Pas d'Utilisation Commerciale - Pas de Modification 4.0 International. 


\title{
Un militantisme aux allures de mission impossible : l'exemple du lobby républicain pro-choice, la Republican Majority for Choice
}

\author{
Françoise Coste
}

1 La discipline de fer imposée par l'administration de George W. Bush sur le Parti républicain donne l'impression que le GOP est un parti très uni. Derrière cette harmonie de surface, la situation est en réalité plus compliquée : il existe au sein du parti un courant qui se distingue par son rejet du conservatisme culturel pur et dur des républicains influencés par la Droite chrétienne. Le principal point d'achoppement entre ces deux branches du parti est la question du droit à l'avortement : il existe en effet des Républicains pro-choice. Certes, il s'agit d'une minorité dans un océan de militants et d'élus fermement pro-life, mais ces Républicains-là existent bel et bien. La principale organisation républicaine pro-choice est la Republican Majority for Choice (RMC).

2 Malgré la taille réduite de cette organisation, elle constitue un sujet d'étude intéressant car ses membres se battent pour une cause dont peu de gens soupçonnent l'existence à l'intérieur du parti de l'éléphant et leur militantisme accumule les dimensions contradictoires qui offrent en réalité un éclairage original sur la politique américaine contemporaine. Il semble d'abord peu logique d'appartenir à un parti dont la lutte contre Roe v. Wade est, dans la période récente, l'un des principes fondamentaux tout en s'investissant dans un lobby tout entièrement dévoué à la sauvegarde du droit à l'avortement aux États-Unis. Il sera donc nécessaire de s'interroger sur la cohérence idéologique du parcours militant des membres de la RMC. De plus, l'analyse des diverses stratégies de lobbying de la RMC et de leur efficacité permettra d'évaluer le poids de ce courant au sein du Parti républicain par rapport à l'influence des lobbies anti-avortement. Plus largement enfin, l'existence d'un groupe comme la RMC pose aussi la question de l'hétérogénéité idéologique au sein des partis politiques 
américains : le Parti démocrate possède lui aussi une frange minoritaire sur la question de l'avortement, une frange pro-life. Que nous apprend le traitement respectif de ces courants minoritaires par les dirigeants républicains et démocrates sur les dynamiques internes des deux partis?

L'apparent paradoxe incarné par les républicains pro-choice est en fait un phénomène récent. Dans les années 1960 et 1970, le GOP n'était pas identifié comme un parti opposé à l'avortement. Ce n'est qu'à partir de 1976 qu'il est devenu officiellement pro-life, et, bien sûr, personne n'a aussi bien représenté ce durcissement idéologique que Ronald Reagan. Sa présidence et ses multiples déclarations hostiles au droit à l'avortement sont à l'origine même de la RMC. En 1984, un groupe de militantes républicaines, écoeurées par le programme pro-life de l'administration Reagan, et menées par des femmes comme Tanya Melich, Barbara Mosbacher, Pauline Harrison et Barbara Gimbel, décidèrent de protester en créant un lobby à la fois républicain et pro-choice, basé à New York : le New York State Republican Family Committee. Ainsi commença une histoire courte mais un peu compliquée : en 1989, le Family Committee a créé son propre PAC (Political Action Committee) lui permettant de verser directement de l'argent aux campagnes de candidats républicains pro-choice. Puis le groupe a changé trois fois de nom: il est devenu la Republican Pro-Choice Alliance en 1995, la Republican Pro-Choice Coalition (RPCC) en 1999, puis la Republican Majority for Choice (RMC) en $2004^{1}$.

Aujourd'hui, la RMC est un lobby national qui, même si son principal bureau reste celui de New York, est présent dans 15 États (Californie, Colorado, Connecticut, Floride, Illinois, Maine, Massachusetts, Michigan, New Jersey, New York, Nevada, Oregon, Pennsylvanie, Texas et Virginie). Il a environ 50000 membres, répartis dans tout le pays. Son objectif est de faire du lobbying pro-choice, du sommet du Parti républicain à sa base, en militant à la fois pour modifier le programme officiel du GOP, en soutenant (surtout financièrement) des candidats républicains pro-choice dans des élections locales et en organisant des campagnes d'informations et de lobbying sur tout ce qui concerne les « women's health issues $»^{2}$.

D'un point de vue idéologique, la RMC est très intéressante à cause de la dichotomie entre son image publique et son message. La réaction la plus fréquente chez les personnes qui découvrent l'existence de républicains pro-choice est la surprise, comme s'il était aberrant d'être à la fois un supporter de Bush et un militant de la cause de l'avortement. Or, nul cas de conscience de ce type n'agite les membres de la RMC. Plus précisément, ses militants ne comprennent pas que l'on puisse s'étonner de leur positionnement idéologique et les considérer comme une anomalie politique, puisqu'ils considèrent qu'être républicain et pro-choice relève en fait de la «chose la plus naturelle du monde ", d'après Lynn Grefe, directrice du lobby de 1995 à 2002. Elle explique ainsi la philosophie de base de la RMC :

Si vous étudiez l'histoire des Républicains, elle est centrée sur la foi en la liberté individuelle. Les gens ont le droit de prendre leur propre décision. Le parti est conservateur sur le plan économique, en faveur d'une intervention limitée de l'Etat. C'est la raison pour laquelle notre organisation dit qu'être pro-choice constitue la véritable position républicaine. Ce n'est pas être pro-avortement, c'est être prochoix. C'est à vous de choisir ce qui se passe dans votre vie privée. [...]

Aujourd'hui, au sein du parti national, on entend des choses comme « Nous sommes pour moins d'Etat, pour un Etat moins intrusif». Et je ne peux pas imaginer un moment où l'Etat peut être plus intrusif que quand il intervient dans la décision d'une femme et d'un homme d'avoir un enfant ${ }^{3}$. 
6 La RMC articule donc son message autour des principes de "liberté individuelle », de "séparation de l'Église et de l'État » et de " responsabilité individuelle », afin que « les choix en matière de reproduction et de contraception demeurent personnels et privés, et non pas dictés par les partis politiques $»^{4}$. En ce sens, l'attachement des militants de la RMC au Parti républicain est compréhensible: depuis son tournant idéologique conservateur entamé dans les années 1960, le GOP considère en effet la notion de big government comme dangereuse pour les libertés individuelles ${ }^{5}$. Pour la RMC, ce refus de l'interventionnisme étatique et cette foi dans le laissez-faire s'appliquent aussi bien aux problèmes économiques qu'aux questions sociales ou médicales comme l'avortement. On peut noter cependant que, malgré son attachement typiquement républicain au concept d'autonomie de l'individu et de small government, et malgré le lien apparemment logique entre la condamnation d'un État qui régule trop l'économie et la condamnation d'un État qui régule la vie privée des citoyens, la RMC soutient quand même les aides publiques pour financer les IVG des femmes les plus pauvres (par le programme Medicaid). L'organisation justifie cette entorse assez évidente à ses professions de foi conservatrices comme le seul moyen de garantir que l'avortement et la contraception demeurent "sûrs, légaux et accessibles à toutes les femmes", «quelques soient leurs revenus $»^{6}$. Le positionnement idéologique de la RMC, s'il peut sembler clair et assumé par ses porte-parole, est donc en réalité plus complexe que ceux-ci ne veulent bien l'admettre. Or de telles contradictions internes peuvent finir par affaiblir l'organisation, en prêtant le flanc aux critiques, en la décrédibilisant aux yeux du reste du parti et en posant la question, on le verra, de la présence à long terme d'un tel courant au sein du Parti républicain.

7 Le militantisme de la Republican Majority for Choice prend plusieurs visages. L'énergie du désespoir pousse les militants à multiplier les actions, à la fois au niveau des États et au niveau fédéral.

8 La plus grande branche locale (« chapter ») de la RMC est dans l'État de New York (avec environ 15000 membres). Les militants new-yorkais sont présents sur plusieurs fronts, en essayant d'appliquer à leur cause les méthodes de lobbying modernes qui ont tant réussi à leurs adversaires idéologiques au sein de la Droite chrétienne. Ainsi, l'organisation multiplie les mailings et les letter campaigns, à la fois vers la population pour éduquer les électeurs au moment des campagnes électorales sur la position prochoice ou pro-life des candidats, mais aussi vers les élus pour tenter de les influencer quand l'assemblée législative de l'État est sur le point de se prononcer sur des lois relatives à l'avortement ou à la contraception. La RMC engage aussi des lobbyistes professionnels qui, accompagnés de responsables du Board de l'organisation ainsi que de militants de base venus de chaque circonscription de l'État, se rendent plusieurs fois par mois à Albany, la capitale. Là, ils rencontrent directement les élus afin de leur démontrer concrètement qu'il existe une masse républicaine pro-choice ${ }^{7}$.

9 Les questions sur lesquelles les Républicains pro-choice ont centré leurs actions à Albany au cours des dernières années leur ont parfois permis de remporter de belles victoires. Lors de la session parlementaire de 1999, (ce qui était alors) la RPCC a par exemple démontré son utilité. Après le meurtre à Buffalo du docteur Slepian par un militant anti-avortement, toute la communauté pro-choice de l'État a réclamé le vote d'une loi empêchant les anti-IVGs de harceler les femmes se rendant dans des cliniques pour y mettre fin à une grossesse. Le texte passa facilement l'Assemblée de l'État, dominée par les Démocrates. Mais le Sénat, dominé par les Républicains, fit preuve de beaucoup de 
réticences : c'est seulement après un lobbying très actif de la part de la RPCC et grâce à ses excellentes relations avec les quelques sénateurs républicains pro-choice d'Albany, que le Sénat finit par voter, le $1^{\text {er }}$ décembre 1999, la loi historique Clinic Access and AntiStalking Act ${ }^{8}$. Sans l'action cruciale de militants pro-choice 'infiltrés' dans la majorité républicaine du Sénat, il n'est pas excessif de penser que les autres lobbys pro-choice (Planned Parenthood, Naral), tous plus ou moins ouvertement liés au Parti démocrate, n'auraient jamais réussi à convaincre à l'assemblée d'État une majorité bipartite.

Plus récemment, en 2003, la RMC a mené une campagne de lobbying fructueuse en parvenant de nouveau à convaincre suffisamment d'élus républicains à Albany de soutenir une autre avancée pour les droits des femmes : l'État de New York a ainsi voté une loi intitulée "Emergency Contraception for Rape Survivors", qui rend accessible des méthodes de contraception d'urgence pour toutes les femmes victimes de viol se rendant aux urgences, même dans les hôpitaux privés et religieux 9 .

11 Logiquement, le lobbying de la RMC au niveau fédéral est d'une autre ampleur. Depuis plusieurs années, l'organisation se bat (sans grand espoir ni réussite) pour faire voter par le Congrès une version nationale de la loi sur la contraception d'urgence pour les victimes de viol ${ }^{10}$. Elle tente également d'être présente sur le front judiciaire, si central dans la lutte pour le droit à l'avortement aux États-Unis. Elle a été tout particulièrement active dans les débats sur la procédure qualifiée de "partial-birth abortion» par les pro-lifers (une méthode utilisée principalement lors des IVGs se déroulant lors du $3^{\text {ème }}$ trimestre de grossesse que les médecins appellent eux « Dilation and Extraction " ou "Dilation and Excavation » - soit D\&X - et consistant à extraire d'abord les pieds du fétus avant d'extraire le crâne, vidé grâce à une succion du cerveau). Les "partial-birth abortions" ont agité le monde politique américain une première fois en 2000, quand la Cour suprême a dû se prononcer sur leur légalité. Or il se trouve que l'homme au cœur de cette tempête judiciaire, LeRoy Carhart, est un membre de la RMC (alors appelée RPCC) ${ }^{11}$. Le docteur Carhart est à l'origine de la plainte déposée contre le ministre de la Justice de l'État du Nebraska quand cet État interdit les avortements de type D\&X. Dans les mois précédant la décision de la Cour suprême Stenberg v. Carhart, qui invalida en 2000 la loi du Nebraska, les responsables de la RPCC ont apporté un soutien constant au docteur Carhart, l'honorant lors de leur dîner de gala annuel à New York au printemps $2000^{12}$.

Quand le président Bush a nommé deux nouveaux juges à la Cour suprême en 2005 et 2006, la RMC a rejoint les nombreux groupes pro-choice, souvent très proches du Parti démocrate, pour tenter de bloquer les nominations des deux juges conservateurs, John Roberts et Samuel Alito. L'organisation craignait surtout que le remplacement de Sandra Day O'Connor, dont la voix avait été cruciale pour obtenir une majorité dans l'arrêt Stenberg v. Carhart, par un juge conservateur n'altère profondément l'équilibre de la Cour sur la question de l'avortement. En 2007, la RMC a poursuivi son action judiciaire, toujours par l'intermédiaire du docteur Carhart, quand celui-ci a attaqué la loi interdisant au niveau fédéral les avortements D\&X, le Partial-Birth Abortion Ban de novembre 2003. Quand, en avril 2007, la Cour a déclaré cette loi constitutionnelle dans sa décision Gonzales v. Carhart, la réaction de la RMC a offert une nouvelle illustration très intéressante de sa schizophrénie idéologique. Au nom des grands principes républicains, elle a en effet fortement condamné cet arrêt qui validait une loi pourtant votée par le Congrès républicain, et signée par un président républicain : «For the first time the Court has stated that it believes the moral decisions of the government take 
precedence over the personal moral decisions of women and families. As Republicans we believe most strongly in individual freedom and limited government intrusion on our personal decisions, including often difficult medical choices ", déclara alors la coprésidente de l'organisation, Jennifer Stockman ${ }^{13}$. Le pan judiciaire de la lutte pour le droit à l'avortement représente ainsi un condensé des contradictions qui sont au cœur du militantisme de la RMC : l'organisation tente d'être présente dans le débat, elle se bat du mieux qu'elle peut avec les quelques armes dont elle dispose, mais elle échoue, terrassée non pas par des opposants politiques mais par d'autres membres de son propre parti, parti dont elle continue malgré tout à citer les principes pour défendre son action.

13 Les militants pro-choice tentent également de se faire entendre lors des conventions nationales du GOP, avec des résultats très variés. Rien par exemple n'a semblé plus démontrer la futilité des efforts des républicains pro-choice que les événements de la convention de juillet 2000 à Philadelphie. Les responsables de ce qui était alors la RPCC élaborèrent un argumentaire qui s'avéra finalement totalement inefficace : ils étaient convaincus que les positions pro-life du parti faisaient fuir de nombreuses électrices vers le Parti démocrate et condamnaient le GOP à des défaites inévitables lors des scrutins nationaux ("Le gender gap se refermera quand les Républicains comme George W. Bush arrêteront de dire aux femmes ce qu'elles peuvent et ne peuvent pas faire " écrivit alors Susan Cullman, la présidente de l'organisation, dans le New York Times) ${ }^{14}$. La RPCC lança une grande campagne de style grass-roots pendant toute l'année 2000, baptisée "Pothole Ahead!», et basée sur l'envoi par les militants aux principaux responsables et élus du GOP de dizaines de milliers de cartes postales jaunes criardes attirant leur attention sur le fait que la position du parti sur l'avortement était « un nid de poule majeur $»$ dans sa route vers la victoire ${ }^{15}$.

14 Mais cette stratégie a piteusement échoué : lors des travaux de la convention, des manipulations des votes du comité chargé de rédiger le programme du parti (comité très influencé par les lobbies de la Droite chrétienne), ainsi que des pressions très fortes exercées par l'équipe Bush sur les délégués pro-choice venus des États du Nord-Est ont empêché la RPCC d'obtenir un minority report reconnaissant l'existence de républicains pro-choice au sein du parti ${ }^{16}$. Autrement dit, à la fin de la saison électorale 2000, le Parti républicain a opposé une fin de non-recevoir à sa minorité favorable au droit à l'avortement.

15 Ceci a logiquement provoqué un électrochoc au sein de l'organisation, qui a alors vu nombre de ses convictions voler en éclat: non seulement sa théorie selon laquelle le positionnement pro-life du GOP l'empêcherait à tout jamais de remporter la Maison Blanche fut invalidée par la victoire de George W. Bush, mais, en plus, les grandes déclarations de fidélité à la philosophie républicaine de ses militants furent bien mises à mal par la désinvolture, voire le mépris, avec lequel le GOP les traita lors de la convention de Philadelphie. Aussi a-t-on pu assister par la suite à de nombreuses évolutions au sein du mouvement.

Le premier changement a eu lieu en mai 2004, quand l'organisation a modifié son nom : la RPCC est devenu la RMC. Cette énième transformation est révélatrice. En effet, à travers les changements de nom successifs de ce lobby, c'est tout un cheminement idéologique et politique que l'on peut identifier. Plus précisément, ces multiples noms trahissent la très délicate position des Républicains pro-choice, à la fois par rapport à leur parti et à l'opinion publique. En 1984, les fondatrices du mouvement optèrent pour 
le nom Family Committee en pensant qu'il était encore possible de se battre avec la Droite Chrétienne sur son propre terrain, celui de la famille. L'une de ses fondatrices, Tanya Melich, justifie ainsi ce choix initial : « Nous avons choisi le nom Family Committee car nous pensions que la clé permettant aux familles d'être fortes dépendait du fait que les personnes aient le droit de choisir. Nous pensions aussi que notre vue tolérante du monde reflétait de façon plus correcte les valeurs familiales de la majorité des Américains $»^{17}$. Mais c'était peine perdue, car il était illusoire de penser pouvoir disputer à la Droite Chrétienne le monopole de l'étiquette des "valeurs familiales". Pire encore, cette stratégie s'avéra contre-productive puisque, lors des premières années d'existence de cette organisation, l'immense majorité des personnes entendant prononcer le nom de Family Committee concluait systématiquement qu'il s'agissait d'un lobby anti-IVG, à cause de la présence du mot «famille», connoté de manière très conservatrice, dans son nom ${ }^{18}$.

17 Aussi le mouvement devint-il la Republican Pro-Choice Alliance en 1995 puis la Republican Pro-Choice Coalition en 1999. Mais l'organisation n'était pas encore au bout de ses peines : ses responsables se sont rendus compte après l'élection de 2000 que de nombreux Républicains effectivement favorables au droit à l'avortement étaient en fait réticents à militer pour la RPCC à cause de la présence du mot «pro-choice» dans son nom. En particulier, dans certaines régions conservatrices, de riches Républicains étaient gênés par le fait que, dans leur entourage, on associait très souvent l'idée d'être pro-choice à celle d'être pro-avortement, même si ce n'est pas exactement la même chose. Aussi futil décidé en 2003 de changer de nom une fois encore.

18 Parallèlement, secoués par les résultats de la convention de Philadelphie, les responsables de la défunte RPCC commanditèrent une grande enquête d'opinion visant à établir de façon fiable le véritable rapport de force au sein du parti sur la question de l'avortement. Ils découvrirent alors que leur raisonnement sur la concordance entre foi républicaine en un Etat limité et soutien au droit à l'avortement était en réalité bien fondé : en effet, $73 \%$ des Républicains interrogés dans tout le pays se sont déclarés, dans ce sondage, « pro-choice ». Les leaders du lobby ont donc décidé d'ajouter à leur nom la notion de "majority ». D'où l'évolution vers Republican Majority For Choice, ainsi justifiée par la directrice du mouvement, Jennifer Wylegala: «Recourir à cette sorte de nom nous garantit d'attirer l'attention. Vous pouvez simplement dire 'Nous somme Républicains et pro-choice', mais quand vous ajoutez le mot 'majorité', vous allez attirer l'attention et les gens vont commencer à poser des questions du style 'Que voulez-vous dire ? Je n'avais jamais considéré la situation en ces termes-là auparavant'. Il s'agit donc d'une manière de provoquer une discussion et de dire qu'une minorité [anti-IVG] a détourné tout le parti $»^{19}$.

19 Cette constante évolution du nom de l'organisation trahit cependant un malaise évident : à travers ces multiples étiquettes apparaît un problème d'identification pour ces républicains pro-choice. La difficulté à trouver un nom satisfaisant et efficace, l'impossibilité même de se réclamer clairement pro-choice et la dimension presque pathétique de ces républicains, complètement ignorés par les instances du parti et s'autoproclamant pourtant majoritaires, montrent bien que leur positionnement idéologique est tellement inhabituel et peu signifiant dans le paysage politique américain, qu'il n'existe en définitive même pas de mots pour le décrire. Ici, le langage de la politique américaine semble nier l'existence, ou plutôt la possibilité même d'existence, de ces militants républicains pro-choice. Le message symbolisé par ce 
problème d'identification est donc que s'il n'y a pas de place pour eux dans l'univers lexical de la politique américaine, il n'y a peut-être finalement pas de place pour eux dans la politique américaine tout court.

Cette situation impossible se reflète parfaitement dans les relations extrêmement compliquées que la RMC entretient avec les dirigeants du GOP.

En général, les Républicains pro-choice sont un peu comme les moutons noirs du parti, des membres de la famille dont on a honte et qu'on essaie d'ignorer. Ainsi, les membres de la RPCC-RMC, lors des grandes messes que sont les conventions présidentielles, se font souvent maltraiter : les délégués de la Droite Chrétienne les traitent de ce qui est sans doute la pire insulte au sein du GOP, « RINOs » (Republicans In Name Only) ${ }^{20}$; on leur refuse le droit d'organiser des conférences de presse (à Houston en 1992) ${ }^{21}$; ou, on l'a $\mathrm{vu}$, on manipule les délibérations de la convention pour tenter d'effacer toute trace de leur participation. En 1996, on pouvait même lire dans le magazine conservateur The Weekly Standard un article consacré aux républicains pro-choice et qui citait sérieusement William F. Buckley pour qui ils seraient favorables à l'IVG car ils considèreraient cette procédure comme « un bon moyen de limiter la population, surtout dans les classes inférieures ", ce qui signifie plus clairement que l'avortement est pour eux « un moyen subtil de limiter le nombre de Noirs nés aux États-Unis ». Le magazine conclut donc en qualifiant les membres de la RPCC de "Social Darwinists " ${ }^{22}$. Ce rejet si intense des républicains pro-choice par leur parti est d'autant plus problématique qu'une organisation comme la RMC ne manque pourtant pas d'atout pour se faire entendre, et ce au plus haut niveau. En particulier parce que ce groupe n'attire pas n'importe quel type de militants. On peut même dire que l'on a là un exemple de militantisme de luxe : les membres de la RMC sont riches, voire très riches (les militantes de la RMC aiment à plaisanter sur le fait que l'outil principal de tout leur lobbying est leurs colliers de perles, sans lesquels elles ne sortent jamais de chez elles) ${ }^{23}$. Pour comprendre quel type de Républicains on trouve à la RMC, quelques exemples : Barbara Mosbacher, l'une des fondatrices, est la sœur de Robert Mosbacher, membre de l'administration Reagan et l'un des plus vieux amis de George H. W. Bush ; Pauline Harrison, une autre fondatrice, est une héritière de la famille DuPont ; Jennifer Stockman, la co-présidente aujourd'hui et la principale figure médiatique du mouvement, est la femme de David Stockman, Directeur du Budget sous Reagan; le chapter du Connecticut compte dans ses rangs Gretchen Johnson et sa fille Scarlett, héritières de la compagnie de produits cosmétiques et ménagers Johnson\&Johnson... La RMC compte aussi quelques stars parmi ses militantes, comme Bo Derek ou Dina Merrill (actrice célèbre dans les années 1950 et, accessoirement, héritière de l'empire financier E.F. Hutton et de la compagnie céréalière Post). Enfin, on trouve dans le "Advisory Committee » de l'organisation des noms qui ont du poids dans le monde républicain, comme ceux de l'ancien président Gerald Ford (jusqu'à sa mort en 2007), la veuve de Barry Goldwater, l'ancien gouverneur de Californie Pete Wilson et même des élus de premier plan aujourd'hui comme le sénateur Arlen Specter de Pennsylvanie ou encore les deux femmes sénateurs du Maine Susan Collins et Olympia Snowe (seules deux autres élues fédérales du GOP sont pro-choice: les Représentantes Nancy Johnson du Connecticut et Judy Biggert de l'Illinois).

De l'aveu même de Pauline Harrison, la RMC est « une organisation élitiste " ${ }^{24}$. C'est vrai qu'on y trouve quasi-exclusivement des Blancs, Protestants (plus quelques Juifs), issus majoritairement du milieu des affaires. Loin d'être anecdotiques, ces détails personnels 
sur les responsables et les membres de la RMC sont d'une importance primordiale pour éclairer l'un des principaux problèmes lié au militantisme des républicains pro-choice: l'origine sociale explique en effet grandement le positionnement républicain de personnes qui sont en réalité très éloignées des préoccupations majeures du GOP. Plus précisément, les principaux animateurs du mouvement appartiennent à la vieille noblesse républicaine, à de très vieilles famille d'élus ou à de très vieilles familles de financiers du parti. Et on a, qui plus est, affaire là à un genre de républicains très singulier : des modérés venant pour la plupart du Nord-Est, complètement étrangers, culturellement et philosophiquement, à la révolution conservatrice reaganienne si influencée par les mouvements religieux et évangéliques présents surtout à l'Ouest et dans le Sud du pays.

Les soutiens et des membres de la RMC constituent donc, en quelque sorte, la mémoire vivante du GOP et appartiennent à une couche sédimentaire très ancienne dans l'évolution idéologique du parti. En ce sens, ce sont les derniers spécimens représentant une période bien particulière de l'histoire de la droite américaine, la dernière preuve que le Parti républicain n'a pas toujours été celui de Ronald Reagan et de George W. Bush. Ceci peut présenter d'incontestables avantages. Par exemple, les responsables de la RMC, par leur niveau socio-économique et nombre de leurs relations familiales, bénéficient d'excellents réseaux qui peuvent ouvrir bien des portes à Washington, même dans les cas que l'on pourrait considérer les plus difficiles. Ainsi, le père de Sam Pryor, l'un des présidents d'honneur du mouvement, était l'un des fondateurs de la défunte compagnie aérienne PanAm. Or il se trouve que Karl Rove (le principal conseiller du président Bush jusqu'en septembre 2007) est un passionné d'aéronautique et de PanAm qui appelle très souvent Sam Pryor, pour parler de son père, ce qui a assuré pendant longtemps un contact régulier entre la RMC et l'un des hommes les plus puissants (et conservateurs) de l'administration Bush ${ }^{25}$.

De même, l'importance des entrées des responsables de la RMC au plus haut niveau du parti a été démontrée en 2004 quand Jennifer Stockman, l'épouse de l'un des républicains les plus influents des années Reagan, a pris la tête de la campagne de lobbying visant à modifier le programme du parti lors de sa convention présidentielle. La visibilité et le statut d'une telle porte-parole ont payé, puisque, contrairement à 2000, le GOP s'est montré plus conciliant en 2004, en acceptant de faire figurer dans son programme officiel un passage prônant une politique de "porte ouverte" et reconnaissant que «le parti [...] respecte et accepte que certains de ses membres puissent avoir des opinions sincères et parfois très différentes ${ }^{26}$. Le mouvement peut donc se targuer d'avoir remporté une victoire symbolique.

Néanmoins, le profil socio-économique si élevé des membres de la RMC soulève en fait de nouvelles questions sur l'efficacité de leur militantisme. Selon les préceptes du pluralisme, la théorie classique utilisée pour étudier l'action des groupes d'intérêts aux États-Unis, tous les intérêts sont libres de s'affronter et cette saine concurrence aboutit à une situation d'équilibre, d'autorégulation et de compromis. Mais la réalité démontre que certains types de groupes d'intérêts sont en fait plus puissants et influents que les autres, un avantage qui découle de leurs plus grandes ressources financières, puisque celles-ci leur permettent de mieux s'organiser et de s'assurer un meilleur accès au champ du politique ${ }^{27}$. Or il semble que le militantisme de la RMC tende à infirmer certains principes du pluralisme. Il pose en particulier d'une nouvelle manière la question du poids de l'argent dans le militantisme moderne. Les lobbies qui ont 
habituellement du mal à se faire entendre aux États-Unis sont ceux qui manquent de moyens pour exister dans le paysage politique, alors que la RMC offre l'exemple assez rare d'un lobby très riche et bien implanté au plus haut niveau du pouvoir politique fédéral, mais qui échoue systématiquement, année après année, à faire avancer sa cause.

Pire encore, on peut même dire que c'est sans doute le considérable accès aux dirigeants du Parti républicain rendu possible par les caractéristiques socioéconomiques des responsables de la RMC qui constitue paradoxalement la principale faiblesse de l'organisation. Parce que les leaders de la RMC appartiennent à la vieille garde du Parti républicain, ils manquent du moyen de pression qui est sans doute le plus utile pour n'importe quel lobby: la possibilité de dire au pouvoir en place « Donnez-nous satisfaction ou bien nous voterons pour quelqu'un d'autre ». En d'autres termes, les dirigeants du Parti républicain ne sont pas très pressés de répondre aux attentes de la RMC car ils savent pertinemment que ces Républicains historiques, ou ces descendants de Républicains historiques, ne quitteront jamais le GOP pour rejoindre le Parti démocrate, par tradition et mémoire familiales. D'ailleurs, la plupart des membres de la RMC le reconnaissent volontiers: avant d'être pro-choice, ils sont républicains, pour des raisons principalement économiques. Les Républicains pro-choice sont des fervents partisans d'un État qui ne s'introduit ni dans la chambre à coucher ni dans le portefeuille des individus. Par conséquent, par conservatisme économique, ils trouveraient aberrant de rejoindre le Parti démocrate, dont ils considèrent l'orientation fiscale comme fondée sur du "gaspillage $»^{28}$. La directrice de la RMC, Jennifer Wylegala, admet honnêtement qu'il n'y a pas eu de rupture spectaculaire avec Bush en 2004 car les militants de l'organisation sont riches et ont donc bénéficié au premier chef des immenses baisses d'impôts voulues par le président lors de son premier mandat ${ }^{29}$. De cette aisance financière des membres de la RMC et de leur fidélité jusqu'ici sans faille aux principes économiques conservateurs du Parti républicain découle donc finalement la situation bien peu ordinaire de cette organisation, celle d'un lobby bénéficiant d'un accès unique aux leaders du Parti républicain sans pour autant exercer sur eux quelque influence que ce soit.

En même temps, il faut noter que cette religion du conservatisme économique pourrait potentiellement poser dans le futur plus de problèmes encore que la seule question de l'avortement. On peut remarquer, en effet, qu'un nouveau type de grogne commence à frapper les membres de la RMC, comme si le véritable conservatisme fiscal n'était plus la chose la mieux partagée entre toutes les branches du GOP. Comme l'explique Jennifer Wylegala, à propos de la dernière élection présidentielle :

Le problème est plus vaste avec Bush : la majorité de nos responsables sont des modérés sur le plan social. [...] Et nombre d'entre eux sont des conservateurs sur le plan fiscal. Et j'en connais beaucoup qui sont en désaccord avec Bush sur tous ces sujets : par exemple, on avait un surplus budgétaire avant [Bush], et maintenant, il a disparu! Notre déficit est astronomique! [...] Donc, je connais beaucoup de nos responsables qui se sont posé des questions au moment de voter en 2004. J'en connais même qui m'ont dit 'Je vais voter pour Mickey, je vais juste écrire Mickey sur le bulletin' ${ }^{\prime 30}$.

Ainsi, si les membres de la RMC apparaissent handicapés dans leur militantisme par leur fidélité quasi-ancestrale au Parti républicain qui les empêche d'exercer une influence réellement efficace sur ses instances nationales, on ne peut s'empêcher de souligner une détérioration des rapports entre la RMC et l'administration Bush. Sur 
beaucoup de questions, Bush semble représenter un test historique pour cette organisation, comme s'il voulait voir jusqu'où il peut aller avant de provoquer la rupture.

Et le résultat assez surprenant de ce test est que si le GOP n'a pas connu de spectaculaires divisions internes sur l'avortement, d'autres sujets sont en train de monter au premier plan et de rendre l'équation des rapports entre RMC et GOP encore plus complexe. On l'a dit, la grogne est fiscale. Mais on peut aussi remarquer que les responsables de la RMC se plaignent également de plus en plus de la politique écologique de Bush (" atroce» selon Jennifer Wylegala), de son opposition au mariage gay et, ce qui est encore plus important à leurs yeux, de son opposition à la recherche sur les cellules souches. Cette question n'est pas directement liée à la lutte pour le droit à l'avortement, mais elle aussi repose sur une problématique où se rejoignent politique et religion, science et idéologie. Le militantisme de la RMC est par conséquent peut-être appelé à se diversifier dans le futur, mais toujours dans un sens opposé à celui des positions des instances dirigeantes du parti.

31 Ces divisions internes grandissantes au sein du Parti républicain sont d'autant plus révélatrices qu'elles constituent sans doute l'une de ses principales différences avec le Parti démocrate. Bien sûr, le Parti démocrate est très largement identifié à la défense du droit à l'avortement. Mais, comme le GOP, il abrite aussi en son sein une frange qui rejette la position officielle du parti sur cette question : il existe aussi des Démocrates pro-life, organisés principalement autour du lobby DFLA (Democrats for Life of America). Or ces dissidents pro-life sont beaucoup plus reconnus par les dirigeants de leur parti que les Républicains pro-choice : non seulement sont-ils plus nombreux à être investis par le Parti démocrate lors des élections, ils sont aussi plus nombreux à siéger au Congrès (on compte actuellement 37 Représentants démocrates pro-life par exemple), mais ils sont en plus beaucoup plus visibles et influents. Ainsi, le démocrate le plus puissant à Washington, le majority leader au Sénat Harry Reid, est-il un Mormon pro-life. La victoire historique des Démocrates sur les Républicains lors des élections de mimandat de novembre 2006 qui ont permis aux Démocrates de reconquérir la majorité aux deux chambres du Congrès s'est en grande partie faite grâce à des candidats modérés qui se réclament de la cause pro-life (comme les sénateurs Bob Casey en Pennsylvanie et Jon Tester dans le Montana). La répartition du pouvoir à l'intérieur du parti entre pro-choicers et pro-lifers est donc bien plus juste chez les Démocrates que chez les Républicains, comme si les premiers étaient bien plus ouverts et prêts à accepter la diversité idéologique qui a toujours caractérisé les partis politiques américains. Cette attitude tolérante face à un courant dissident est d'autant plus remarquable chez les Démocrates qu'elle correspond à un intérêt électoral bien compris. Les candidats démocrates pro-life permettent à leur parti de l'emporter dans des États d'habitude acquis aux Parti républicain (comme le Montana pour reprendre l'exemple de Jon Tester). Alors que dans le cas du GOP, le parti, par son rejet quasisystématique des dissidents pro-choice, se prive de militants et d'élus aux profils pourtant originaux et potentiellement fructueux sur le plan électoral : ainsi, deux élus proches par le passé de la RMC, Rudy Giuliani (ancien maire de New York) et de Mitt Romney (ancien gouverneur du Massachusetts), ont été nettement battus lors des primaires de 2008, la base républicaine ne leur pardonnant pas leur modération sur la question du droit à l'avortement (même si Romney a fait campagne en reniant son soutien passé au camp pro-choice, son soudain revirement n'a convaincu personne et a 
confirmé son statut de persona non grata parmi la base très conservatrice du parti). Dans cette optique, le principal intérêt du militantisme d'un groupe comme la Republican Majority for Choice réside dans sa fonction de baromètre quant à la dynamique interne du Parti républicain : un parti si hostile à une minorité finalement si peu dangereuse et rebelle n'est peut-être pas un parti en aussi bonne santé idéologique que sa façade d'unanimité pendant les années Bush n'a pu le laisser penser.

\section{BIBLIOGRAPHIE}

Entretiens avec l'auteur

Lynn Grefe, New York City, 7 janvier 2000

Jennifer Wylegala, New York City, 2 mars 2005

Publications RPCC/RMC

Republican Pro-Choice Coalition, GOP Voice for Choice, RPCC Newsletter (Décembre 1999).

Republican Pro-Choice Coalition, If You Are A Republican, If You Are Pro-Choice, Then Join the Republican Pro-Choice Coalition Today !, RPCC Brochure (2000).

Republican Majority for Choice, Voice for Choice, RMC Newsletter (août 2000).

Republican Pro-Choice Coalition, A Summary of the Pro-Choice Efforts at the Convention, RPCC Newsletter (18 août 2000).

Republican Majority for Choice, Voice for Choice, RMC Newsletter (automne 2004).

Republican Majority for Choice. RMC's Statement on the Supreme Court Ruling on Federal Abortion Ban Cases (18 avril 2007). 20/09/2007. https://gopchoice.electionmall.name/E-PressRelease/

displaycontent.asp?a=5C5E5E\&z=5D

Sources secondaires

B. Drummond, Ayres Jr., « Political Briefing : Republicans Facing An Abortion Battle », The New York Times (26 mars 2000).

Carlson, Tucker, « What Pro-Choice Republicans Believe », The Weekly Standard (24 juin 1996).

Cullman, Susan R., « Closing the Gender Gap », The New York Times (1er avril 2000).

Gerring, John, Party Ideologies in America, 1828-1996 (Cambridge : Cambridge University Press, 1998).

GOP Republican Platform 2000, Renewing America's Purpose Together (Philadelphia Republican National Convention, 2000).

Lowi, Theodore J. et Benjamin Ginsberg, American Government, Freedom and Power (New York : W. W. Norton \& Company, $5^{\text {ème }}$ édition, 1998).

Melich, Tanya, The Republican War against Women (New York : Bantam, 1996). 
Nardi, Christine, Rogue Elephants : The RPCC and the Politics of Abortion (Sarah Lawrence College : Masters of Arts Degree, 2000).

Stan, Adele M. « Inside the Republican Pro-Choice Coalition » Salon.com (29 juillet 2000).

\section{NOTES}

1. Le changement de nom de 1999 s'explique par la fusion de l'organisation avec un autre groupe républicain pro-choice, la Republican Coalition for Choice, créé lui en 1989 par une ancienne cadre très haut placée au sein des instances nationales du parti, Mary Dent Crisp (Nardi, 21-25).

2. Interview de l'auteur avec Jennifer Wylegala, 2 mars 2005.

3. Interview de l'auteur avec Lynn Grefe, 7 janvier 2000. Toutes les traductions sont celles de la rédactrice.

4. Brochure RPCC, Archives RPCC, 2000.

5. Gerring, 125.

6. Brochure RPCC, Archives RPCC, 2000.

7. Interview avec Jennifer Wylegala, 2 mars 2005.

8. GOP Voice for Choice, RPCC Newsletter, décembre 1999.

9. Interview avec Jennifer Wylegala, 2 mars 2005.

10. Interview avec Jennifer Wylegala, 2 mars 2005.

11. http://dir.salon.com/story/politics/feature/2000/07/29/merrill/

12. Voice for Choice, RPCC Newsletter, août 2000.

13. https://gopchoice.electionmall.name/E-PressRelease/displaycontent.asp? $a=5 C 5 E 5 E \& z=5 D$

14. Cullman, New York Times, 1er avril 2000, A14.

15. B. Drummond Ayres Jr., New York Times, 26 mars 2000, 32.

16. A Summary of the Pro-Choice Efforts at the Convention, RPCC Newsletter, 18 aout 2000. La seule concession du parti fut d'accorder un paragraphe à la RPCC à la toute fin des annexes du programme officiel adopté en 2000. Les instances du GOP y admettent que l'organisation a effectivement déposé une motion devant le Platform Committee réclamant de voir son existence mentionnée dans le texte (les irrégularités du vote lors de ce 'débat' sont aussi implicitement citées, sans qu'un observateur n'ayant pas assisté directement à ces événements ne puisse vraiment en réaliser la teneur) (Renewing America's Purpose Together, GOP Republican Platform 2000, 2000).

17. Melich, 185-186.

18. Nardi, 31-32.

19. Interview avec l'auteur, 2 mars 2005.

20. Nardi, 17-18.

21. Melich, 262.

22. Carlson, The Weekly Standard 24 juin 1996, 30-33.

23. Interview avec Lynn Grefe, 7 janvier 2000.

24. Nardi 33.

25. Interview avec Jennifer Wylegala, 2 mars 2005.

26. Voice for Choice, RMC Newsletter, automne 2004.

27. Lowi and Ginsberg, 519-520.

28. Nardi, 42. 
29. Interview avec Jennifer Wylegala, 2 mars 2005.

30. Interview avec Jennifer Wylegala, 2 mars 2005.

\section{RÉSUMÉS}

The active lobbying of the Republican Majority for Choice proves the conventional wisdom about the monolithic pro-life nature of the contemporary Republican Party wrong. The RMC is a wealthy and growing organization which has concretely helped the advancement of abortion and reproductive rights, especially at the state level. But it has remained almost completely powerless to influence the national leaders of the GOP, primarily because of the historical association between most of its top-ranking members and the Republican establishment, which prevents any credible electoral threat. Yet, an undeniable rift is widening between RMC activists and the Bush administration, as the president's acute social conservatism and reckless spending violate the basic tenets of the social moderation and fiscal conservatism of pro-choice Republicans.

\section{INDEX}

Mots-clés : Avortement, parti républicain, idéologie conservatrice, Cour suprême, républicains modérés

Keywords : lobbying, Abortion, Republican Party, conservative ideology, Supreme Court, moderate Republicans

\section{AUTEUR}

\section{FRANÇOISE COSTE}

Maître de Conférences, Université Toulouse 2, francoste@hotmail.com. 